\title{
Avaliação preliminar das cargas difusas de origem rural, em uma sub-bacia do Rio Jaguari, SP
}

\author{
Maria T. C. Mansor ${ }^{1}$, José Teixeira Filho ${ }^{1} \&$ Denis M. Roston ${ }^{1}$
}

\begin{abstract}
RESUMO
Dados oficiais de concentração de nutrientes e vazão do Rio Jaguari, foram utilizados na avaliação das contribuições difusas, com origem rural, às águas superficiais de uma sub-bacia do Rio Jaguari, SP. Modelos de cargas médias diárias de $\mathrm{N}_{\text {total }}$ e $\mathrm{P}_{\text {total }}$ foram gerados a partir de regressão estatística aplicada a dados de campo obtidos entre 1999 e 2002. A validação dos modelos foi feita utilizando-se dados de campo obtidos entre 1996 e 1998. O modelo mostrou que, em média, $68,2 \%$ das cargas anuais de $\mathrm{N}_{\text {total }}$ e $77,3 \%$ das cargas anuais de $\mathrm{P}_{\text {total }}$ foram transportados pela sub-bacia no período chuvoso. Partindo-se de cargas de base diárias de 1706,5 $\mathrm{kg} \mathrm{N}$ total e de 165,7 kg $\mathrm{P}_{\text {total, }}$ determinou-se que 55,1 e $69,4 \%$ das cargas anuais totais de $\mathrm{N}_{\text {total }}$ e $\mathrm{P}_{\text {total, }}$ respectivamente, transportadas pela sub-bacia, provieram de fontes difusas. As taxas de exportação de nutrientes por carga difusa, obtidas pela aplicação do modelo, foram iguais a $230 \mathrm{~kg} \mathrm{~N}_{\text {total }} \mathrm{km}^{-2}$ ano-1 e $40 \mathrm{~kg} \mathrm{P}_{\text {total }} \mathrm{km}^{-2}$ ano $^{-1}$. Concluiu-se que o controle das fontes difusas de $\mathrm{P}_{\text {total, }}$ a partir de áreas rurais, deverá diminuir as concentrações deste nutriente no Rio Jaguari.
\end{abstract}

Palavras-chave: qualidade da água, modelagem, fósforo total, nitrogênio total, fontes não pontuais de poluição

\section{Preliminary assessment of diffused loads from rural areas in a sub-basin of the Jaguari River, SP, Brazil}

\begin{abstract}
Official water quality and flow data from Jaguari River were used to assess the diffused loads of nutrients from rural areas into surface waters. Mathematical models of mean daily loads of total nitrogen (TN) and total phosphorus (TP) were generated through the application of simple statistical procedures to field data collected from 1999 to 2002. The models obtained were validated with field data collected from 1996 to 1998. The application of the model showed that, on average, $68.2 \%$ of the TN annual loads and $77.3 \%$ of the TP annual loads were transported through the sub-basin during the raining season. Assuming the mean baseflow loads as $1706.5 \mathrm{~kg} \mathrm{TN} \mathrm{d}^{-1}$ and $165.7 \mathrm{~kg} \mathrm{TP} \mathrm{d}^{-1}$, the annual diffused loads obtained - primarily from rural sources - were equivalent to 55.1 and $69.4 \%$ of the total annual loads of TN and TP transported through the sub - basin, respectively. The mean export coefficients from diffused loads for TN and TP were equal to 230 and $40 \mathrm{~kg} \mathrm{~km}^{-2}$ year-1, respectively. In may be concluded that controlling the diffused loads of TP from rural areas should decrease the high concentrations observed.
\end{abstract}

Key words: water quality, modeling, total phosphorus, total nitrogen, non point source pollution 


\section{INTRODUÇÃO}

A percepção e a importância relativa das cargas difusas de nutrientes às águas superficiais, têm aumentado a medida em que se procede à identificação e ao subseqüente controle das cargas pontuais, como documentam as recentes publicações científicas sobre o assunto (Carpenter et al., 1998; Macleod \& Haygarth, 2003; Witt \& Behrendt, 1999; Tanik et al., 1999; Silva \& Porto, 2003). As cargas pontuais de nutrientes estão associadas a qualquer meio perceptível, confinado ou desviado de transporte de poluentes - especialmente de águas residuárias domésticas e industriais - para as águas superficiais. Diferente destas, as cargas difusas de nutrientes são aquelas geradas em áreas extensas e que, associadas às precipitações pluviométricas, chegam às águas superficiais de forma intermitente, em especial a partir de áreas rurais. A poluição por cargas difusas de nutrientes pode ter natureza urbana, rural ou atmosférica. Em áreas urbanas, a poluição difusa tem composição complexa - de metais e óleos a sólidos - constituindo-se numa fonte de poluição tanto maior quanto mais deficiente for a coleta de esgotos, ou mesmo a limpeza pública; já na área rural, a poluição difusa é devida, em grande parte, à drenagem pluviométrica de solos agrícolas e ao fluxo de retorno da irrigação, sendo associada aos sedimentos (carreados quando há erosão do solo), aos nutrientes (nitrogênio e fósforo) e aos defensivos agrícolas. A drenagem das precipitações em áreas de pecuária é associada, ainda, aos resíduos da criação animal - nutrientes, matéria orgânica e coliformes. A deposição atmoférica de nutrientes - especialmente nitrogênio - provenientes de emanações industriais e queimadas de matas/cana-de açúcar, e o arraste de partículas e gases da atmosfera por águas pluviais, também são considerados poluição difusa (Macleod \& Haygarth, 2003; Dalcanale, 2001; CETESB, 2003; Martinelli et al., 2002; Novotny, 1999).

De acordo com Macleod \& Haygarth (2003), mensurar a importância de fontes pontuais e difusas de nutrientes na escala de uma bacia hidrográfica, envolve ainda muitas incertezas. Este fato advém da poluição difusa ser de difícil quantificação, uma vez que depende da interação de diversos fatores, como a intensidade e duração das precipitações, o tipo de solo, a fisiografia do terreno e as formas de uso do solo (Diogo et al., 2003). Carpenter et al. (1998) estudaram as fontes difusas de nutrientes nos Estados Unidos e concluíram que 82 e $84 \%$ dos descartes de $\mathrm{N}_{\text {total }}$ e $\mathrm{P}_{\text {total }}$, respectivamente, nas águas superficiais, provêm de fontes difusas, majoritariamente (acima de 90\%) de áreas rurais. Macleod \& Haygarth (2003) compilaram vários estudos feitos na Europa sobre a importância de fontes difusas de fósforo com origem agrícola, e concluíram que as referidas fontes contribuem com 29 a $60 \%$ da carga total observada nas águas superficiais daquelas bacias, porém esses estudos são específicos e, no Brasil, enquanto os efluentes domésticos e industriais pontuais têm características conhecidas, os agrícolas difusos não estão ainda quantificados e estudos a este respeito são incipientes.

Novotny \& Olem (1993) classificam os modelos de simulação de cargas difusas em níveis que vão desde procedimen- tos estatísticos simples e cargas unitárias sem interação com processos físico-químicos até modelos contínuos sofisticados. Enquanto Eiger et al. (1999) e Diogo et al. (2003) se basearam em procedimentos estatísticos simples e cargas unitárias, respectivamente, para avaliar cargas difusas de nutrientes, Dalcanale (2001) e Silva \& Porto (2003) as simularam, aplicando modelos sofisticados desenvolvidos por agências governamentais norte-americanas, que demandam grande quantidade de dados. Em todos esses casos, três deles no Brasil, os trabalhos resultaram em análises consideradas preliminares, quer pela pouca disponibilidade de dados de monitoramento para as bacias estudadas, quer por utilização de índices, coeficientes e padrões desenvolvidos para países de clima temperado. Neste último aspecto, Pessoa et al. (1997) compilaram os modelos matemáticos e simuladores sofisticados mais citados por pesquisadores agropecuários na década de 90 - dos quais vários aplicáveis à análise e quantificação de cargas difusas - tendo sido todos, sem exceção, desenvolvidos em países de clima temperado.

O Rio Jaguari possui nascentes no Estado de Minas Gerais e foz no encontro com o Rio Atibaia, no Estado de São Paulo, onde ocorre a formação do Rio Piracicaba. Ele flui de leste para oeste e seu principal afluente é o Rio Camanducaia. Dentro do Estado de São Paulo, o Rio Jaguari está inserido no $5^{\circ}$ grupo de Unidades de Gerenciamento de Recursos Hídricos, formado pelas bacias hidrográficas dos Rios Piracicaba, Capivari e Jundiaí - UGRHI-PCJ. A bacia do Rio Piracicaba é considerada modelo de "bacia desenvolvida", sendo responsável por quase 10\% do PIB e do Valor de Transformação Industrial do Brasil. Ela é qualificada com o principal vetor de desconcentração industrial metropolitana de São Paulo, porém tem ocupação bastante heterogênea, com algumas regiões tipicamente voltadas para o setor industrial e outras para o setor agrícola, com produção intensiva. Na bacia hidrográfica do Rio Piracicaba, as culturas de cana-de-açúcar, citros e as pastagens, são as atividades agrícolas mais significativas em termos econômicos e em extensão. Os Rios da bacia hidrográfica do Rio Piracicaba abastecem, hoje, 42 municípios - 33 por captação em mananciais de superfície - e recebem efluentes de cerca de 194 indústrias, além de efluentes domésticos de 40 municípios, dos quais somente 13 possuem algum sistema de tratamento de esgotos (Martinelli et al., 2002; CETESB, 2003; Del Grande et al., 2003; CBH-PCJ, 2001).

A bacia hidrográfica do Rio Jaguari está voltada para o setor agrícola e, apesar de apresentar sinais visíveis de princípio de degradação, comparativamente com os demais mananciais da bacia hidrográfica do Rio Piracicaba, é ainda a mais preservada (CBH-PCJ, 2001). Este princípio de degradação está bem retratado em CETESB (2004) que apresentou, no ano de 2003, uma concentração média de $\mathrm{P}_{\text {total }}$ de $0,793 \mathrm{mg} \mathrm{L}^{-1}$ no ponto JAGR02800 (Rio Jaguari na captação de Limeira, 4,5 km à montante da confluência com o Rio Atibaia), enquanto a média dos últimos 10 anos foi igual a $0,146 \mathrm{mg} \mathrm{L}^{-1}$. No mesmo ponto, a concentração média de nitrato observada em 2003 foi igual a $1,77 \mathrm{mg} \mathrm{L}^{-1}$ e a média dos últimos 10 anos igual a $0,83 \mathrm{mg} \mathrm{L}^{-1}$. Por fim, a média anual do Índice de Estado Trófico - que considera as concentrações de $\mathrm{P}_{\text {total }}$ e clorofila- $a$ indicou estar o curso d’água em estado eutrófico. 
A garantia da qualidade e quantidade da água do Rio Jaguari - que assegure a reversão de cerca de $30 \mathrm{~m}^{3} \mathrm{~s}^{-1}$ de água para abastecimento da Região Metropolitana de São Paulo, pelo Sistema Cantareira, e o abastecimento dos municípios da sua própria bacia, que drena uma região com mais de 400 mil habitantes - passa, necessariamente, pelo combate às cargas difusas de nutrientes com origem rural, uma vez que esta bacia tem ocupação agrícola. Para efeito de enquadramento na Resolução CONAMA 20, o Rio Jaguari é considerado classe 1 nos trechos à montante da barragem do Sistema Cantareira e classe 2 no trecho à jusante do Sistema. A ocupação majoritariamente rural torna a bacia hidrográfica do Rio Jaguari adequada para determinação preliminar da dimensão das contribuições difusas rurais de nutrientes às águas superficiais na região.

O objetivo deste estudo foi quantificar as contribuições difusas rurais de $\mathrm{N}_{\text {total }}$ e $\mathrm{P}_{\text {total }}$ às águas superficiais de uma sub-bacia do Rio Jaguari.

\section{MATERIAL E MÉTODOS}

A bacia hidrográfica do Rio Jaguari (Figura 1) tem área total de $4.320 \mathrm{~km}^{2}$, dos quais 70,4\% se localizam no Estado de São Paulo. Em território paulista, 98\% da área da bacia se encontram na zona rural, com uso e ocupação assim distribuídos: 14,2\% em agricultura temporária, 15,3\% em agricultura perene, 3,7\% em cobertura vegetal natural, 3,5\% em reflorestamentos e 61,3\% em pastagens e campos antrópicos (CBH-PCJ, 2001).

A maioria dos solos da bacia do Rio Piracicaba apresenta baixo pH e baixa fertilidade natural. Há predominância de solos argilosos, de dois tipos: Latossolo Vermelho-Amare- lo e Argissolos. A bacia hidrográfica do Rio Piracicaba se caracteriza por invernos secos (abril-agosto) e verões chuvosos (outubro-março). A precipitação média anual varia de 1300 a $1800 \mathrm{~mm}$; as médias mensais indicam que os meses menos chuvosos são julho e agosto e as maiores precipitações ocorrem em dezembro e janeiro. A sazonalidade da vazão segue aquela da precipitação; a vazão média do Rio Jaguari, entre 1947 e 1992, foi de $54 \mathrm{~m}^{3} \mathrm{~s}^{-1}$ (Martinelli et al., 2002).

Para desenvolvimento deste estudo tomou-se, como seção de controle no Rio Jaguari, o posto fluviométrico 4D-001 do DAEE-SP, denominado Usina Estér, com Latitude 22 39' 26”" Sul e Longitude $47^{\circ} 12^{\prime}$ 53”' Oeste (Figura 1). A área de drenagem da sub-bacia de estudo, delimitada pelo posto 4D-001, é igual a 3.394 km² (78,6\% da área total da bacia hidrográfica do Rio Jaguari). Os dados de vazão média diária do Rio Jaguari foram obtidos junto ao DAEE- SP (São Paulo, 2003), que os calcula por curva-chave a partir de leituras linimétricas diárias no posto 4D-001.

Os dados de qualidade de água aqui utilizados, referemse à estação JAGR02800 no Rio Jaguari - Latitude $22^{\circ} 40^{\prime}$ Sul e Longitude $47^{\circ} 12^{\prime}$ Oeste - mantida pela CETESB. Os dados utilizados foram amostrados entre 1996 e 2002 pela CETESB (1996; 1997; 1998; 1999; 2000; 2001; 2003), por coleta simples, com freqüência bimensal (janeiro, março, maio, julho, setembro e novembro), sendo os parâmetros aqui considerados: $\mathrm{N}_{\text {total }}, \mathrm{P}_{\text {total }}$ e resíduos não filtráveis. Os procedimentos laboratoriais utilizados pela CETESB estão descritos em CETESB (1996; 1997; 1998; 1999; 2000; 2001; 2003).

A seção do Rio Jaguari de amostragem de água (ponto JAGR02800 da CETESB) não é coincidente geograficamente com a seção de monitoramento de vazões (posto 4D-001 do

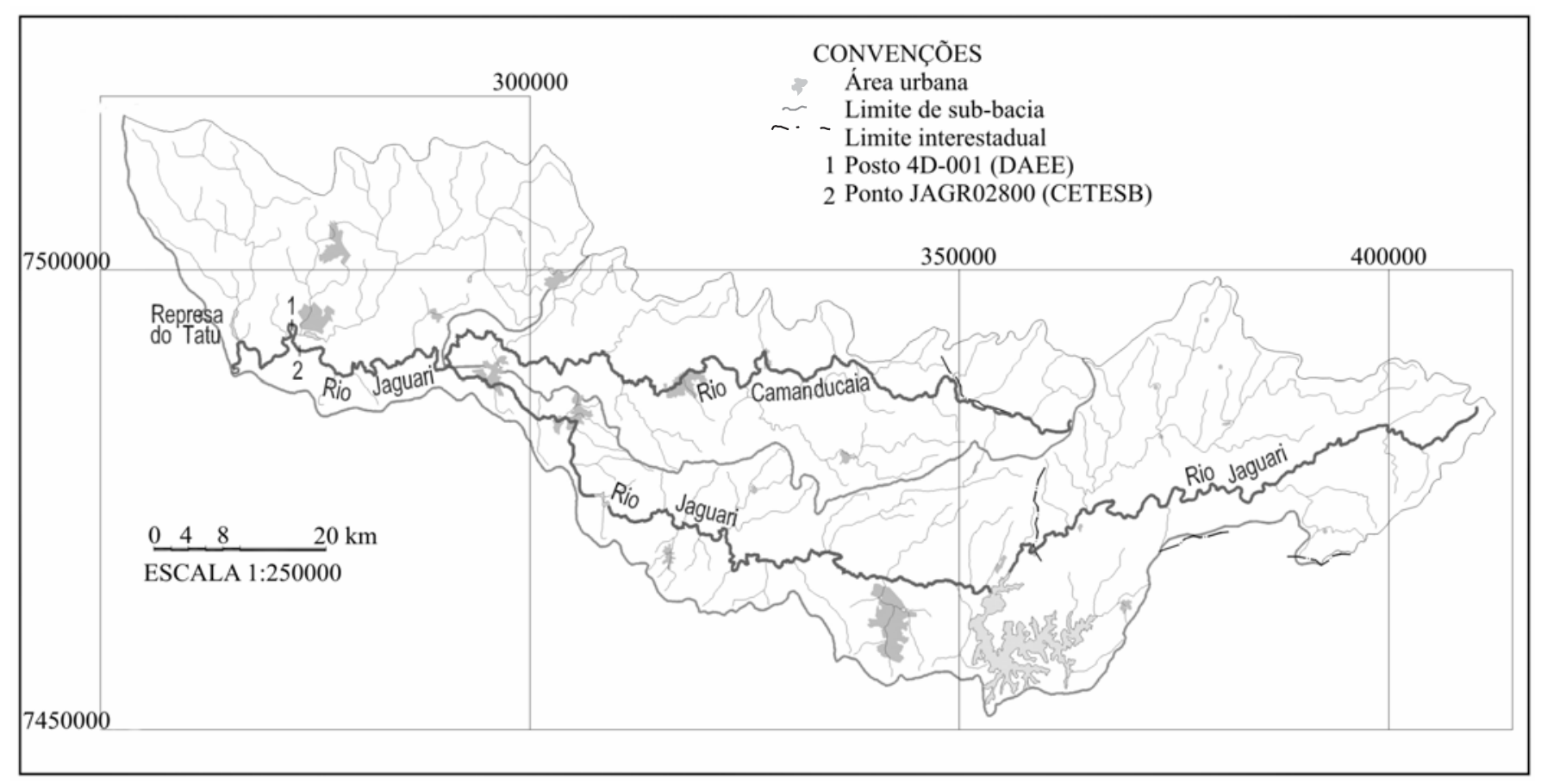

Figura 1. Bacia hidrográfica do Rio Jaguari com localização do posto 4D-001 (DAEE) e ponto JAGR02800 (CETESB) 
DAEE) considerada neste estudo, porém as duas seções são próximas o suficiente para se considerar que os dados de qualidade sejam representativos na seção de monitoramento de vazão.

\section{Avaliação estatística dos dados}

Inicialmente, os dados de campo de vazão do posto 4D-001 e de concentração de $\mathrm{N}_{\text {total }}$ e $\mathrm{P}_{\text {total }}$ do posto JAGR2800, obtidos no período de 01/99 a 09/02, foram usados no cálculo de cargas diárias totais de $\mathrm{N}_{\text {total }}$ e $\mathrm{P}_{\text {total }}$. Uma regressão estatística foi então aplicada para relacionar as cargas diárias de $\mathrm{N}_{\text {total }}$ e $\mathrm{P}_{\text {total }}$ transportadas pelo Rio Jaguari e as correspondentes vazões médias diárias. As dimensões da sub-bacia em estudo permitiram fazer-se este tipo de avaliação por regressão estatística.

Em uma etapa posterior do estudo, os dados de campo de vazão do posto 4D-001 e de concentração de $\mathrm{N}_{\text {total }}$ e $\mathrm{P}_{\text {total }}$ do ponto JAGR2800, obtidos no período de 01/96 a 11/98, foram usados no cálculo de cargas diárias totais de $\mathrm{N}_{\text {total }}$ e $\mathrm{P}_{\text {to- }}$ tal. Tais cargas diárias totais de $\mathrm{N}_{\text {total }}$ e $\mathrm{P}_{\text {total }}$ foram então comparadas com aquelas correspondentes, geradas pela regressão estatística, a partir da vazão.

Por receber grande aporte de efluentes domésticos e industriais pontuais e grande aporte de efluentes agrícolas difusos (CBH-PCJ, 2001; Agujaro \& Isaac, 2002), assumiu-se que as cargas diárias de base de $\mathrm{N}_{\text {total }}$ e $\mathrm{P}_{\text {total }}$, transportadas pela sub-bacia do Rio Jaguari, tivessem origem preponderantemente doméstica e industrial (Macleod \& Haygarth, 2003). Assim, estabeleceu-se que as cargas diárias de base de $\mathrm{N}_{\text {total }}$ e $\mathrm{P}_{\text {total }}$ fossem iguais às médias das cargas diárias calculadas pelos modelos de regressão para os meses de julho e agosto dos anos 1996 a 2002. Nesses meses, a contribuição difusa de nutrientes, proveniente das regiões rurais, foi considerada ínfima, pela diminuição do escoamento superficial provocado por precipitações na sub-bacia. As cargas de base foram, portanto, consideradas pontuais, de origem urbana.

A partir daí, as cargas difusas de $\mathrm{N}_{\text {total }}$ e $\mathrm{P}_{\text {total }}$ foram obtidas pela diferença entre as cargas diárias totais, estimadas para o período de 01/96 a 09/02 utilizando-se os respectivos modelos de regressão e as cargas médias de base.

\section{RESULTADOS E DISCUSSÃO}

O valor médio da vazão medida no posto 4D-001, no período de 1996 a 2002, foi de $41,3 \mathrm{~m}^{3} \mathrm{~s}^{-1}$. Os valores médios das concentrações de $\mathrm{N}_{\text {total }}$ e $\mathrm{P}_{\text {total }}$ medidas no ponto JAGR02800, no mesmo período, foram de 1,684 e $0,159 \mathrm{mg} \mathrm{L}^{-1}$, respectivamente. No caso do $\mathrm{N}_{\text {total }}$, não se registraram concentrações mais altas que o teor máximo permitido pela Resolução CONAMA 20 para o Rio Jaguari, porém no caso do $\mathrm{P}_{\text {total }}$, ocorreu preponderância de concentrações acima do teor máximo estabelecido.

\section{Cargas diárias de $\mathbf{N}_{\text {total }}$}

Ajustaram-se os dados de campo obtidos de 01/99 a 09/ 02 à relação polinomial $y=-0,09 x^{2}+107,44 x$, com $R^{2}=0,89$ (Figura 2A), para a carga diária de $\mathrm{N}_{\text {total }}$ transportada. No ajuste dessa equação, dois pontos extremos de concentração foram desconsiderados:

- $\quad 0,35 \mathrm{mg} \mathrm{L}^{-1}$, a mais baixa concentração de $\mathrm{N}_{\text {total }}$ registrada de 1996 a 2002, observada em 01/02 associada a uma vazão relativamente alta $\left(115,9 \mathrm{~m}^{3}\right)$

- 4,5 mg L ${ }^{-1}$, a mais alta concentração de $\mathrm{N}_{\text {total }}$ registrada de 1996 a 2002, observada em 05/02

A concentração de $\mathrm{N}_{\text {total }}$ variou entre 0,35 e $4,5 \mathrm{mg} \mathrm{L}^{-1}$ no período de 01/99 a 09/02, com média de $1,75 \mathrm{mg} \mathrm{L}^{-1}$; as maiores concentrações foram observadas em época de estiagem. A vazão variou entre 10,2 e 214,4 $\mathrm{m}^{3} \mathrm{~s}^{-1}$, com média de 42,4 $\mathrm{m}^{3} \mathrm{~s}^{-1}$; a sazonalidade da vazão seguiu aquela da precipitação.

Fez-se a validação do modelo matemático ajustado (Figura 2B) com valores de carga de $\mathrm{N}_{\text {total }}$ calculados a partir de dados de campo obtidos entre 01/96 e 11/98. A análise de regressão mostrou que existe correlação significativa entre os dados de campo e os estimados com o uso da equação de regressão ajustada ( $\mathrm{p}<0,05$ para o coeficiente angular), com $\mathrm{R}^{2}$ igual a 0,83 . Verificou-se, neste caso, que o modelo matemático apresenta tendência a subestimar as cargas de $\mathrm{N}_{\text {total }}$. As

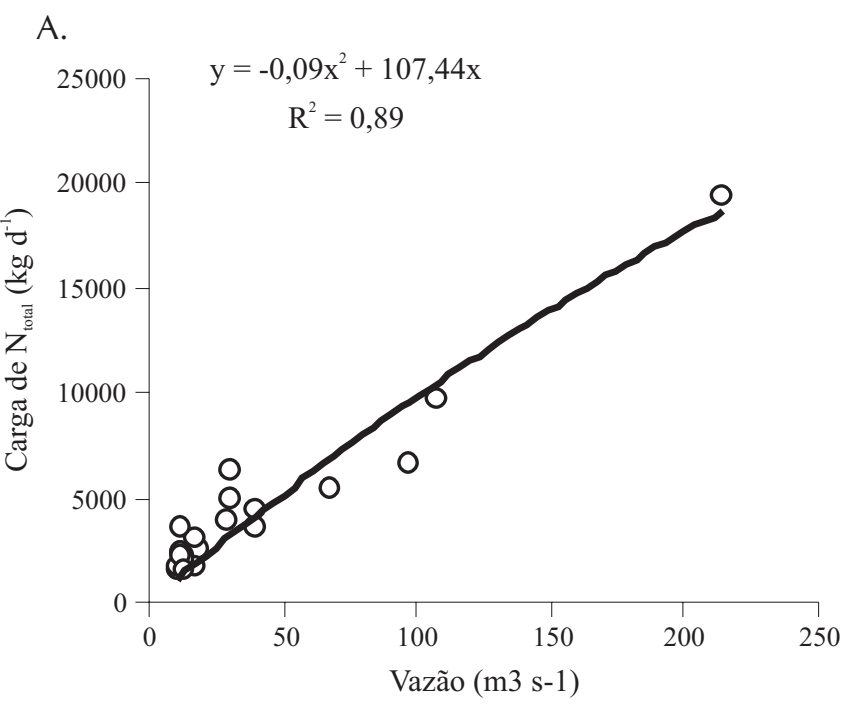

B.

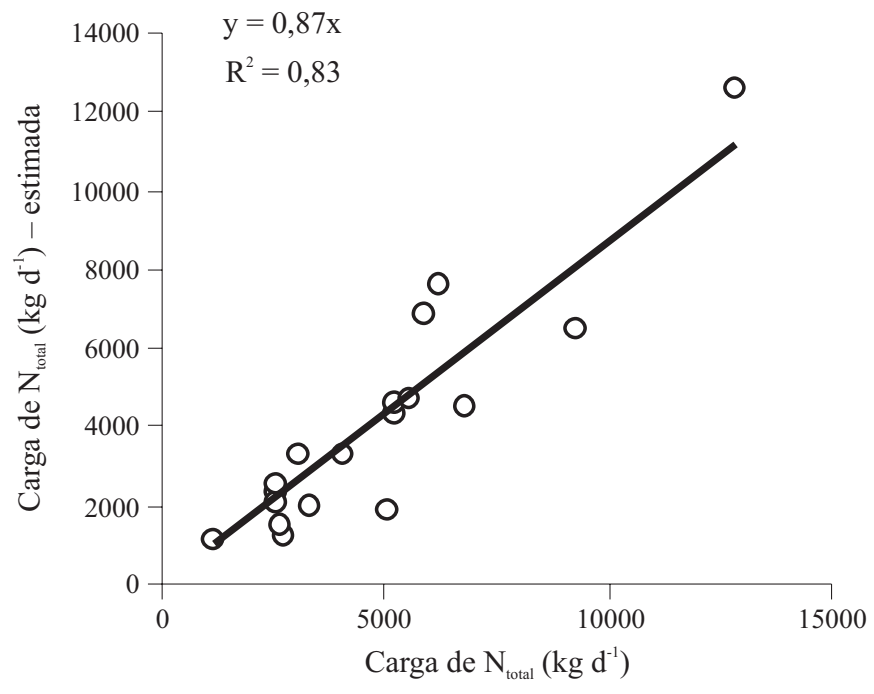

Figura 2. Modelo de carga diária de $\mathrm{N}_{\text {total }}$ (A) e validação do modelo matemático ajustado (B) utilizando-se dados CETESB 


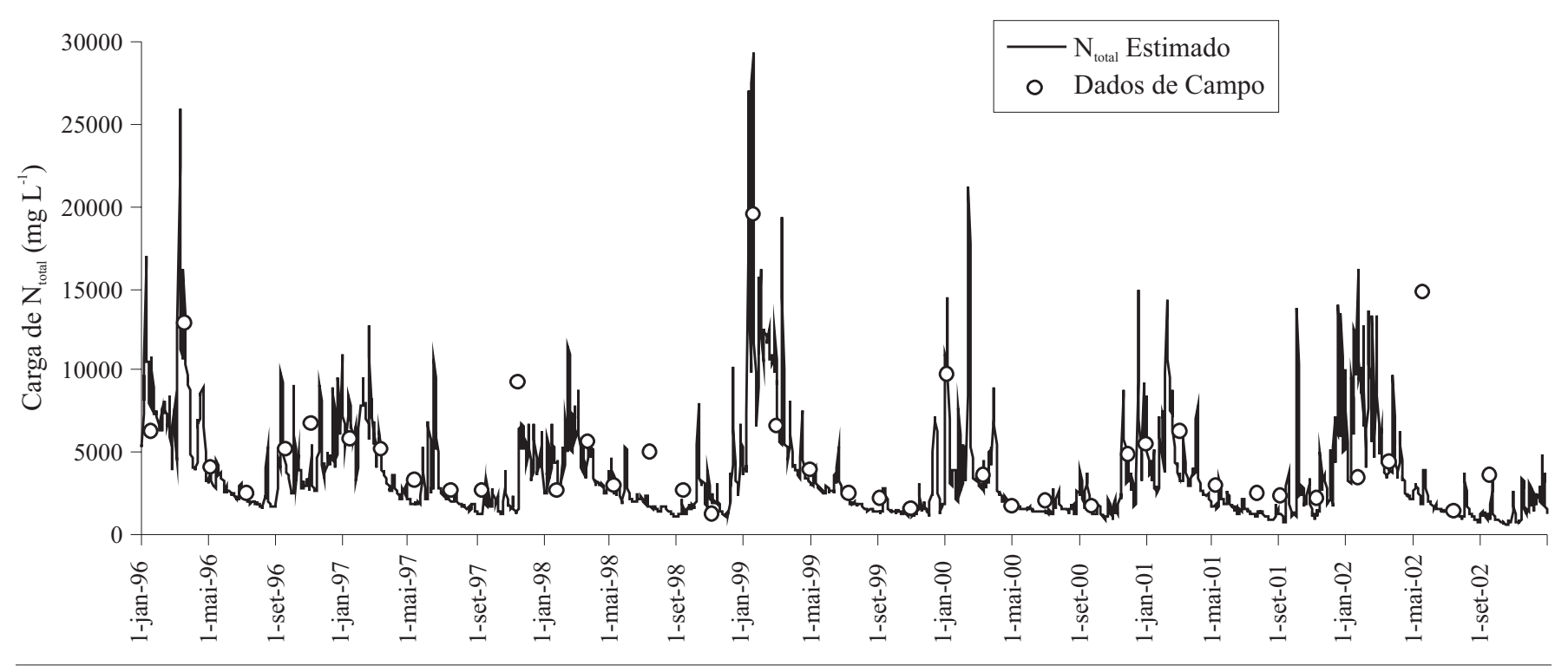

Figura 3. Carga média diária de $\mathrm{N}_{\text {total }}$ valores observados e estimados

maiores diferenças entre o valor de carga de $\mathrm{N}_{\text {total }}$ medida em campo e o valor calculado pelo modelo matemático - acima de $40 \%$ em números absolutos - foram observadas quando se registraram as mais altas concentrações de $\mathrm{N}_{\text {total }},\left(>2 \mathrm{mg} \mathrm{L}^{-1}\right)$.

A concentração de $\mathrm{N}_{\text {total }}$ variou entre 0,95 e 3,21 $\mathrm{mg} \mathrm{L}^{-1}$ no período de 01/96 a 11/98, com média de 1,61 $\mathrm{mg} \mathrm{L}^{-1}$; as maiores concentrações foram constatadas em época de estiagem. A vazão variou entre 11 e $133 \mathrm{~m}^{3} \mathrm{~s}^{-1}$, com média de $40 \mathrm{~m}^{3} \mathrm{~s}^{-1}$; a sazonalidade da vazão seguiu aquela da precipitação.

A Figura 3 mostra que o ajuste entre os valores de carga média diária de $\mathrm{N}_{\text {total }}$, observados e simulados ao longo do tempo, foi muito bom e, visualmente, pôde-se inferir que a carga média diária de $\mathrm{N}_{\text {total }}$ foi significativamente maior na época chuvosa e a carga difusa de $\mathrm{N}_{\text {total }}$ deve ter sido maior que a correspondente carga de base no período.

Avaliou-se, por fim, a dependência entre a magnitude das cargas de $\mathrm{N}_{\text {total }}$ e a magnitude das cargas de sólidos suspensos totais $\left(\mathrm{SS}_{\text {totais }}\right)$, por verificação da existência de relação estatística entre os dados de campo obtidos no período de 01/ 96 a 09/02. Obteve-se $R^{2}$ igual a 0,58 para a relação linear, o que mostrou existir correlação entre as respectivas cargas, ou seja, dependência entre as cargas de $\mathrm{N}_{\text {total }}$ geradas na subbacia e o escoamento superficial causado pelas precipitações pluviométricas.

\section{Cargas diárias de $\mathbf{P}_{\text {total }}$}

Ajustaram-se os dados de campo obtidos de 01/99 a 09/ 02 à relação polinomial $\mathrm{y}=0,09 \mathrm{x}^{2}+8,63 \mathrm{x}$, com $\mathrm{R}^{2}=0,97$ (Figura 4A), para a carga diária de $\mathrm{P}_{\text {total }}$ transportada. No ajuste desta equação, não se desconsideraram pontos extremos.

A concentração de $\mathrm{P}_{\text {total }}$ variou entre 0,008 e $0,539 \mathrm{mg} \mathrm{L}^{-1}$ no período de 01/99 a 09/02, com média de 0,188 $\mathrm{mg} \mathrm{L}^{-1}$; não se observou tendência entre o valor da concentração e a época de cheia ou estiagem.

Fez-se a validação do modelo matemático ajustado (Figura 4B) com valores de carga de $\mathrm{P}_{\text {total }}$ calculados a partir

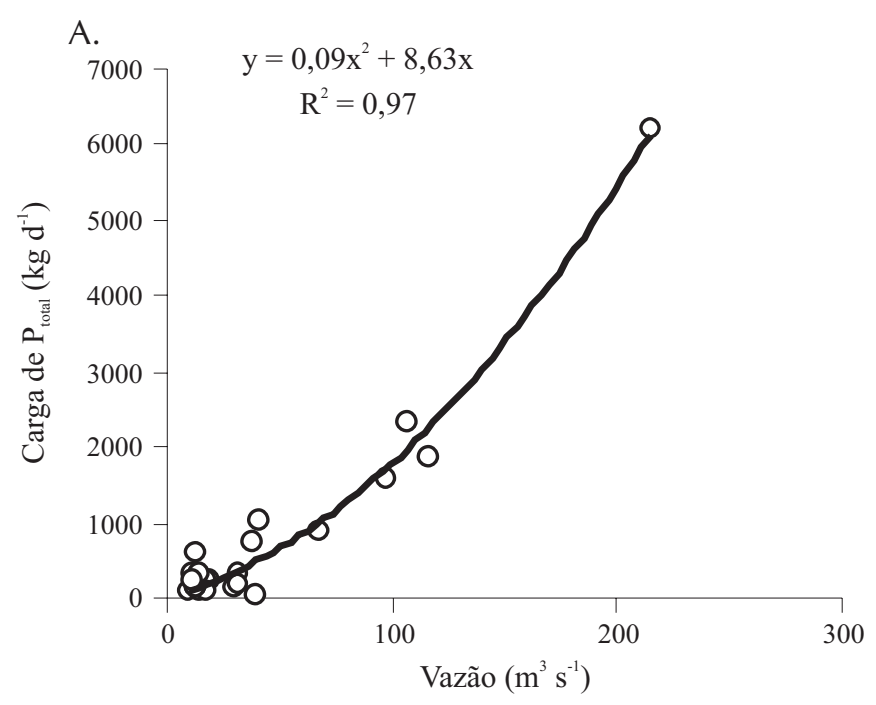

B.

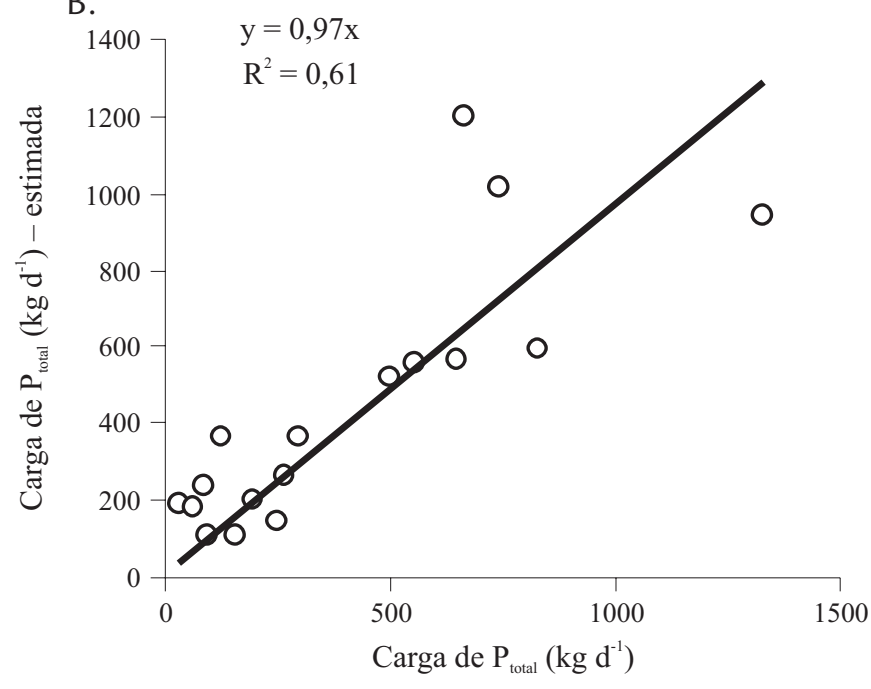

Figura 4. Modelo de carga diária de $\mathrm{P}_{\text {total }}$ (A) e Validação do modelo matemático ajustado (B) utilizando-se dadas CETESB 
de dados de campo obtidos entre 01/96 e 11/98. A análise de regressão, feita desconsiderando-se a carga referente à maior vazão registrada no período $\left(133 \mathrm{~m}^{3} \mathrm{~s}^{-1}\right)$, mostrou haver correlação significativa entre os dados de campo e estimados ( $\mathrm{p}<0,05$ para o coeficiente angular), com $\mathrm{R}^{2}$ igual a 0,61. Verificou-se, neste caso, que o modelo matemático apresentou tendência a subestimar as cargas de $\mathrm{P}_{\text {total }}$, tal como ocorrido com o $\mathrm{N}_{\text {total }}$.

As maiores diferenças entre os valores de carga de $\mathrm{P}_{\text {total }}$ medida em campo e os estimados com o uso do modelo matemático, acima de $42 \%$ em números absolutos, foram observadas nas datas em que se registraram as mais baixas concentrações de $\mathrm{P}_{\text {total }}\left(<0,45 \mathrm{mg} \mathrm{L}^{-1}\right)$, ou as mais altas vazões (76 e $\left.133 \mathrm{~m}^{3} \mathrm{~s}^{-1}\right)$. A concentração de $\mathrm{P}_{\text {total }}$ variou entre 0,021 e 0,239 $\mathrm{mg} \mathrm{L}^{-1}$ no período de 01/96 a 11/98, com média de 0,121 mg L-1; não se observou, porém, tendência entre o valor da concentração e a época de cheia ou estiagem.

A Figura 5 mostra que o ajuste entre os valores de carga média diária de $\mathrm{P}_{\text {total }}$, observados e simulados ao longo do tempo, foi muito bom e, visualmente, pôde-se inferir que a carga média diária de $\mathrm{P}_{\text {total }}$ foi significativamente maior na época chuvosa e a carga difusa de $\mathrm{P}_{\text {total }}$ deve ter sido maior que a correspondente carga de base no período, tal como registrado também para o $\mathrm{N}_{\text {total }}$.
Avaliou-se, por fim, a dependência entre a magnitude das cargas de $\mathrm{P}_{\text {total }}$ e a magnitude das cargas de $\mathrm{SS}_{\text {totais }}$ por verificação da existência de relação estatística entre os dados de campo obtidos no período de 01/96 a 09/02. Obteve-se $\mathrm{R}^{2}$ igual a 0,95 para a relação linear, o que mostrou existir forte correlação entre as respectivas cargas, ou seja, dependência entre as cargas de $\mathrm{P}_{\text {total }}$ geradas na sub-bacia e o escoamento superficial causado pelas precipitações. A tendência de adsorção do fósforo às partículas do solo, em oposição à maior mobilidade do nitrogênio no solo, poderia explicar o resultado mais significativo obtido para o $\mathrm{P}_{\text {total}}$, em relação ao $\mathrm{N}_{\text {total }}$.

\section{Contribuições difusas de nutrientes na sub-bacia}

Obteve-se, para $\mathrm{N}_{\text {total }}$, carga média de base de $1706,5 \mathrm{~kg} \mathrm{dia}^{-1}$ e, para $\mathrm{P}_{\text {total }}$, carga média de base de $165,7 \mathrm{~kg} \mathrm{dia}^{-1}$.

A aplicação do modelo matemático ajustado para estimativa da carga diária de $\mathrm{N}_{\text {total }}$ entre 01/96 e 09/02, mostrou que $31,8 \%$ da carga anual foram transportados nos meses de estiagem (abr-set), enquanto 68,2\% o foram nos meses chuvosos (out-mar). No caso do $\mathrm{P}_{\text {total }}, 22,7 \%$ da carga anual foram transportados nos meses de estiagem, enquanto $77,3 \%$ o foram nos meses chuvosos. Esses dados demonstram a importância das cargas difusas de nutrientes na sub-bacia do Rio Jaguari. A associação entre as cargas médias diárias de

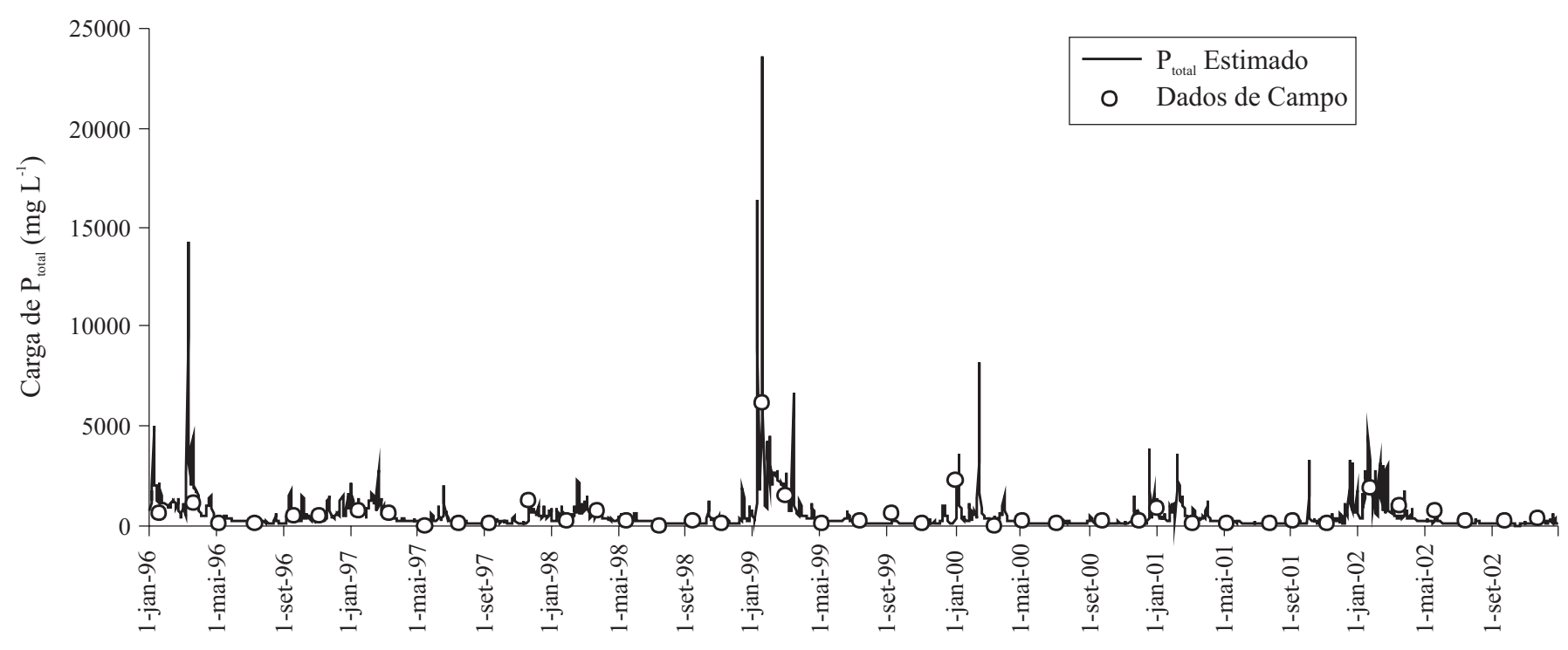

Figura 5. Carga média diária de $\mathrm{P}_{\text {total}}$, valores observados e estimados

Tabela 1. Cargas médias anuais pontuais e difusas de $\mathrm{N}_{\text {total }}$ e $\mathrm{P}_{\text {total }}\left(\mathrm{N}_{\text {total }}-\mathrm{P}_{\text {total }}\right)$

\begin{tabular}{|c|c|c|c|c|c|c|}
\hline Ano & $\begin{array}{l}\text { Volume total (4D-001) } \\
\qquad\left(10^{9} \mathrm{~m}^{3}\right)\end{array}$ & $\begin{array}{l}\text { Carga pontual urbana } \\
\text { (t ano-1) }\end{array}$ & $\begin{array}{l}\text { Carga difusa rural } \\
\qquad\left(\mathrm{t} \mathrm{ano}^{-1}\right)\end{array}$ & $\begin{array}{l}\text { Carga total anual } \\
\qquad\left(\mathrm{t} \mathrm{ano}^{-1}\right)\end{array}$ & $\begin{array}{l}\text { Carga difusa rural } \\
\% \text { do total anual }\end{array}$ & $\begin{array}{c}\text { Coeficientes } \\
\text { exportação rural } \\
\left(\mathrm{kg} \mathrm{km}^{-2} \text { ano-1) }\right.\end{array}$ \\
\hline 1996 & 1,60 & $624,4-60,6$ & $1261,1-243$ & $1885,5-303,6$ & $66,9-80,0$ & $380-70$ \\
\hline 1997 & 1,11 & $608,6-58,8$ & $716,6-111,5$ & $1325,2-170,3$ & $54,1-65,5$ & $220-30$ \\
\hline 1998 & 0,95 & $598,7-57,7$ & $538,3-79,4$ & $1137,0-137,1$ & $47,3-57,9$ & $160-20$ \\
\hline 1999 & 1,46 & $592,3-56,9$ & $1031,2-283,3$ & $1623,5-340,2$ & $63,5-83,3$ & $310-90$ \\
\hline 2000 & 0,97 & $585,4-56,1$ & $553,6-104,0$ & $1139,0-160,1$ & $48,6-65,0$ & $160-30$ \\
\hline 2001 & 0,99 & $567,3-54,3$ & $606,3-102,5$ & $1173,6-156,8$ & $51,7-65,4$ & $180-30$ \\
\hline 2002 & 0,99 & $542,0-51,6$ & $621,9-113,4$ & $1163,9-165,0$ & $53,4-68,7$ & $190-30$ \\
\hline Média & 1,15 & $588,4-56,6$ & $761,3-148,2$ & $1349,7-204,7$ & $55,1-69,4$ & $230-40$ \\
\hline
\end{tabular}


$\mathrm{N}_{\text {total }}$ e $\mathrm{P}_{\text {total }}$ medidas em campo, e as cargas de $\mathrm{SS}_{\text {totais }}$ correspondentes mostraram, ainda, que ambas devem ter o mesmo mecanismo gerador para as situações de cheia, conforme também observaram Eiger et al. (1999) para a bacia hidrográfica do Rio do Peixe. Desta forma, assumiu-se que as contribuições difusas fossem geradas pelo escoamento superficial proveniente de áreas rurais, considerado uso e ocupação da sub-bacia em estudo. Calcularam-se, também, os coeficientes de exportação referentes às cargas difusas de origem rural. Na Tabela 1 encontram-se esses resultados, obtidos ano a ano.

Observou-se, nos resultados apresentados na Tabela 1, a existência de uma forte correlação entre os dados de descarga total (no ponto 4D-001) e carga difusa rural (em \%), tanto para $\mathrm{N}_{\text {total }}$ quanto para $\mathrm{P}_{\text {total }}$. Em ambos os casos, notou-se tendência ao aumento da carga difusa total anual transportada com o aumento da descarga total anual, o que implicou em coeficientes de exportação mais altos para as maiores vazões totais anuais. A partir desses resultados, calculou-se a razão entre a carga média anual de origem rural e urbana; para o $\mathrm{N}_{\text {total }}$, obteve-se uma razão de 1,3 e, para o $\mathrm{P}_{\text {total}}$, uma razão de 2,6. Eiger et al. (1999) estimaram as cargas potenciais urbanas de nutrientes existentes no esgoto doméstico da bacia hidrográfica do Rio do Peixe, SP, e observaram que estas foram cerca de dez vezes menores que as cargas difusas anuais estimadas por modelagem. As contribuições mais equilibradas obtidas na bacia hidrográfica do Rio Jaguari, tanto para $\mathrm{N}_{\text {total }}$ quanto para $\mathrm{P}_{\text {total }}$, podem ser devidas ao fato do número de habitantes da bacia do Rio Jaguari ser cerca de 20 vezes maior que aquele da bacia hidrográfica do Rio do Peixe. Ressalta-se, porém, que em números absolutos, Eiger et al. (1999) obtiveram carga difusa anual para o $\mathrm{N}_{\text {total }}$ de $752 \mathrm{t}$, equivalente, portanto, àquela aqui obtida, produzida, contudo, em uma área 5,7 vezes menor. No caso do $\mathrm{P}_{\text {total }}$, a carga obtida por Eiger et al. (1999), de 42,6 t anuais, foi 3,5 vezes menor que a obtida no Rio Jaguari. Partindo desses altos valores, Eiger et al. (1999) calcularam as cargas difusas específicas para $\mathrm{N}_{\text {total }}$ e $\mathrm{P}_{\text {total }}$, obtendo 1270 e $70 \mathrm{~kg} \mathrm{~km}^{-2}$ ano-1, respectivamente, enquanto as obtidas neste estudo foram iguais a 230 e $40 \mathrm{~kg} \mathrm{~km}^{-2} \mathrm{ano}^{-1}$, respectivamente. Diogo et al. (2003) compilaram taxas de exportação de $\mathrm{N}_{\text {total }}$ e $\mathrm{P}_{\text {total }}$ por carga difusa para diferentes classes de uso do solo, a saber: 500 e $100 \mathrm{~kg} \mathrm{~km}^{-2} \mathrm{ano}^{-1}$ em áreas agrícolas com culturas anuais, 390 e $70 \mathrm{~kg} \mathrm{~km}^{-2}$ ano-1 em áreas agrícolas heterogêneas, 270 e $30 \mathrm{~kg} \mathrm{~km}^{-2}$ ano $^{-1}$ em áreas agrícolas com culturas permanentes ou zonas com vegetação arbustiva ou herbácea, 200 e $100 \mathrm{~kg} \mathrm{~km}^{-2}$ ano-1 $^{-1}$ em florestas e 150 e $90 \mathrm{~kg} \mathrm{~km}^{-2}$ ano $^{-1}$ em pastagens. Kalff (2002) compilou valores típicos de coeficientes de exportação de $\mathrm{N}_{\text {total }}$ e $\mathrm{P}_{\text {total }} \mathrm{e}$ apresentou, para bacias ocupadas por agricultura, em países de clima temperado, valores de 660 e $20 \mathrm{~kg} \mathrm{~km}^{-2}$ ano-1, com intervalo de variação de $500-1000 \mathrm{~kg} \mathrm{~km}^{-2}$ ano-1 e 5$50 \mathrm{~kg} \mathrm{~km}^{-2}$ ano $^{-1}$, respectivamente.

Os coeficientes obtidos neste estudo estão próximos àqueles apresentados por Diogo et al. (2003) para áreas agrícolas com culturas permanentes ou zonas com vegetação ar-

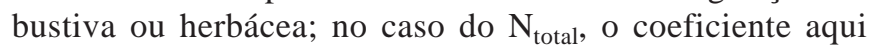

obtido foi menor e, no caso do $\mathrm{P}_{\text {total }}$, equivalente. Esses coeficientes foram calculados para uma área total de $3.394 \mathrm{~km}^{2}$ (dos quais $62,7 \%$ se situam em território paulista e o restante em Minas Gerais) cujos principais usos são pastagens e campos antrópicos.

\section{Relação $\mathbf{N}_{\text {total }} / \mathbf{P}_{\text {total }}$}

As cargas diárias de $\mathrm{N}_{\text {total }}$ e $\mathrm{P}_{\text {total }}$ estimadas foram usadas no cálculo da variação temporal da relação $\mathrm{N}_{\text {total }} / \mathrm{P}_{\text {total }}$ molar. $\mathrm{O}$ valor médio calculado para a relação $\mathrm{N}_{\text {total }} / \mathrm{P}_{\text {total }}$, foi igual a 20 (variando entre 6,7 e 24,6), enquanto os dados de campo produziram valor médio de 29 (variando entre 4,1 e 76,1). No cálculo do valor médio de campo, desconsideraram-se 3 situações, nas quais os valores extremos de um nutriente não foram acompanhados por valor extremo do outro. Os casos de concentração muito baixa de fósforo dificilmente condizem com as atuais condições de degradação ambiental do Rio Jaguari. Kalff (2002) apresentou uma relação $\mathrm{N}_{\text {total }} / \mathrm{P}_{\text {total }}$ molar típica de 29 para as bacias ocupadas por agricultura em clima temperado, valor coincidente com a média dos dados de campo obtida neste estudo. A variação temporal da relação $\mathrm{N}_{\text {total }}$ / $\mathrm{P}_{\text {total }}$ indicou tendência de aumento em períodos secos, conforme já haviam observado Eiger et al. (1999) para o Rio do Peixe.

Por fim, a definição do nutriente limitante deste sistema foi feita considerando-se duas referências, a saber: (1) Salas \& Martino (2001), que estabeleceram que relação $\mathrm{N}_{\text {total }} / \mathrm{P}_{\text {total }}$ molar maior que 20 (ou mássica maior que 9) indica limitação por fósforo em lagos tropicais; (2) Kalff (2002), que afirma que razão $\mathrm{N}_{\text {total }} / \mathrm{P}_{\text {total }}$ de suprimento molar maior que 16 reflete limitação por fósforo e uma razão de suprimento molar menor que 10 reflete limitação por nitrogênio, enquanto valores intermediários indicam crescimento aproximadamente balanceado. Portanto, o fósforo deve ser o nutriente limitante para o crescimento do fitoplancton na sub-bacia hidrográfica do Rio Jaguari, o que coincide com o caso estudado por Eiger et al. (1999).

Não há, hoje, um controle específico de fontes difusas de nutrientes na UGRHI-PCJ, que dimensione a importância relativa dessas fontes em relação às pontuais, conforme se fez de forma incipiente neste estudo. Este controle exigiria intenso monitoramento ambiental e levantamentos de campo e, para viabilizá-lo, é necessário lançar mão de modelos de simulação matemática. Dever-se-ia, portanto, prosseguir estudos desta natureza, a fim de se criar condições de utilização de modelos mais complexos e de se definir coeficientes de exportação que retratem as condições locais. Isto possibilitaria a aplicação efetiva desses modelos nos processos de tomada de decisão para a gestão de bacias hidrográficas e para procedimentos de licenciamento ambiental. A caracterização e quantificação da poluição difusa de origem rural poderiam auxiliar na implantação da cobrança pelo despejo de resíduos, na recuperação das matas ciliares e na conservação do solo. Ressalta-se que existe previsão de implantação de estações de tratamento primário e secundário de esgotos domésticos na bacia do PCJ, porém o tratamento terciário, que removeria os 
nutrientes dessas fontes pontuais, não está, a princípio, contemplado, por ser ainda economicamente inviável. Por outro lado, o combate às fontes difusas de nutrientes provenientes de áreas agrícolas, definidas aqui como de maior importância que as pontuais, se faz prioritário nesta subbacia, porém ainda não estão contemplados nas políticas públicas. O manejo mais conservativo e sustentável do solo agrícola ajudaria a controlar as fontes difusas rurais de nutrientes, em especial aquelas de $\mathrm{P}_{\text {total}}$, em geral responsáveis pela eutrofização dos corpos de água.

\section{CONCLUSÕES}

1. Os cálculos das cargas médias diárias de $\mathrm{N}_{\text {total }}$ e $\mathrm{P}_{\text {to- }}$ tal obtidos por regressão estatística, a partir de dados de vazão média diária obtidos no campo, mostraram-se apropriados para avaliação preliminar da relação entre carga pontual e difusa da sub-bacia hidrográfica do Rio Jaguari.

2. Existe importância da poluição difusa de origem rural na degradação das águas superficiais nesta bacia, que se encontram em acelerado processo de eutrofização.

3. A dependência entre a magnitude das cargas de nutrientes e a magnitude do escoamento superficial gerado pela chuva, ficou caracterizada.

4. A diminuição das altas concentrações de nutrientes observadas no Rio Jaguari deve passar, necessariamente, pelo combate às cargas difusas, em especial de $\mathrm{P}_{\text {total}}$, de origem rural.

\section{AgRADECIMENTOS}

Os autores agradecem à FAPESP e ao Convênio Internacional CAPES/Fulbright.

\section{LITERATURA CITADA}

Agujaro, L. F.; Isaac, R. L. Ocorrência de cianobactérias potencialmente tóxicas nas bacias dos Rios Piracicaba, Capivari e Jundiaí - Estado de São Paulo, Brasil - e avaliação de seus corpos d’água em relação à eutrofização. In: Congreso Interamericano de Ingenieria Sanitária y Ambiental, 28, 2002, Cancun, México. Resumo expandido... México: AIDS, 2002. CD Rom.

Carpenter, S. R.; Caraco, N. F.; Correll, D. L.; Howarth, R. W.; Sharpley, A. N.; Smith, V. H. Nonpoint pollution of surface waters with phosphorus and nitrogen. Ecological Applications, Washington, v.8, n.3, p.559-568, 1998.

CBH-PCJ. Plano de bacia hidrográfica 2000-2003: relatório final - fase 3. Piracicaba: Coplaenge/FEHIDRO. 2001. 240p. http://www.comitepcj.sp.gov.br/CT-EA/Diag_CT-EA.htm > 27 Out.2004
CETESB. Relatório de qualidade das águas interiores do Estado de São Paulo. São Paulo: Secretaria do Meio Ambiente. 102p, 1996. Série relatórios

CETESB. Relatório de qualidade das águas interiores do Estado de São Paulo. São Paulo: Secretaria do Meio Ambiente. 217p, 1997. Série relatórios

CETESB. Relatório de qualidade das águas interiores do Estado de São Paulo. São Paulo: Secretaria do Meio Ambiente. 266p, 1998. Série relatórios

CETESB. Relatório de qualidade das águas interiores do Estado de São Paulo. São Paulo: Secretaria do Meio Ambiente. 1999. Relatório interno

CETESB. Relatório de qualidade das águas interiores do Estado de São Paulo. São Paulo: Secretaria do Meio Ambiente. 2000. Relatório interno

CETESB. Relatório de qualidade das águas interiores do Estado de São Paulo. 2001. São Paulo: Secretaria do Meio Ambiente. v.1. Série relatórios < http://www.cetesb.sp.gov.br/Agua/ agua_geral.asp >, 06 Jun.2003.

CETESB. Relatório de qualidade das águas interiores do Estado de São Paulo. 2003. São Paulo: Secretaria do Meio Ambiente. v.1, 273p. Série relatórios < http://www.cetesb.sp.gov.br/Agua/ agua_geral.asp >, 22 out.2004.

Dalcanale, F. Simulação de cargas difusas em bacias rurais. São Paulo: Escola Politécnica/USP, 2001. 110p. Dissertação Mestrado

Del Grande, M.; Rezende, M. O. O.; Rocha, O.Distribuição de compostos organoclorados nas águas e sedimentos da bacia do Rio Piracicaba - Brasil. Química Nova, São Paulo, v.26, n.5, p.678-686, 2003.

Diogo, P. A.; Coelho, P. S.; Almeida, M. C.; Mateus, N. S.; Rodrigues, A. C. Estimativa de cargas difusas com origem agrícola na bacia hidrográfica do Rio Degebe. In: Simpósio de Hidráulica e Recursos Hídricos dos Países de Língua Oficial Portuguesa, 6, 2003, Cabo Verde. Anais...Cabo Verde: INGRH, APRH, ABRH e AMCT, 2003. p.135-146. CD-Rom

Eiger, S.; Salati, E.; Petracco, P.; Salati, E.; Marcondes, D. S. Modelagem de cargas de nutrientes para a bacia hidrográfica do Rio do Peixe, SP. In: Simpósio Brasileiro de Recursos Hídricos, 13, 1999, Belo Horizonte. Anais... São Paulo: ABRH, 1999. CD-Rom

Kalff, J. Limnology: Inland water ecosystems. New Jersey: Prentice-Hall, 2002. p.341-348.

Macleod C.; Haygarth, P. A review of the significance of non-point source agricultural phosphorus to surface water. Scope Newsletter, Devon, UK, n.51, p.1-10, 2003.

Martinelli, L. A.; Victoria, R. L.; Ferraz, E. S.; Camargo, P. B.; Moreira, M. Z.; Krusche, A. V.; Moraes, J. M.; Ballester, M. V.; Lara, L. L. S.; Bernardes, M. C.; Ometto, J. P. H. B.; Cerri, C. E. Hydrology and water quality in the Piracicaba River basin, São Paulo State, Brazil. In: McClain, M.E. (eds). The ecohydrology of South American Rivers and Wetlands. Unietd Kingdon: IAHS, 2002. n.6, p.159177. Special publication

Novotny, V. Difuse pollution from agriculture. Water Science and Technology, v.39, n.3, p.1-13, 1999.

Novotny, V.; Olem, H. Water quality: Prevention, identification and management of diffuse pollution. New York: Van Nostrand Reinhold, 1993. 458p. 
Pessoa, M. C. P. Y.; Luchiari, A. J.; Fernandes, E. N.; Lima, M. A. Principais modelos e simuladores utilizados para análise de impactos ambientais das atividades agrícolas. Jaguariuna: Embrapa-CNPMA, 1997. 80p.

Salas, H.; Martino, P. Metodologias simplificadas para la evaluación de eutroficacion en lagos cálidos tropicales. Lima: CEPIS. OPS/OMS, 2001. 60p.

São Paulo. SIGRH. Regionalização hidrológica no Estado de São Paulo. <http://www.sigrh.sp.gov.br/sigrh/basecon/bancodedados/flu/flu.htm> 14abr.2003.
Silva, A. S.; Porto, M. F. A. A utilização do modelo WinHSPF no estudo das cargas difusas de poluição da bacia do ribeirão da Estiva, SP. In: Simpósio Brasileiro de Recursos Hídricos, 15, 2003, Curitiba. Anais...São Paulo: ABRH, 2003. CD-Rom.

Tanik, A.; Beler Baykal, B.; Gonenc, I. E. The impact of agricultural pollutants in six drinking water reservoirs. Water Science and Technology, v.40, n.2, p.11-17, 1999.

Witt, M.; Behrendt, H. Nitrogen and phosphorus emissions from soil to surface water in the Rhine and Elbe basins. Water Science and Technology, v.39, n.12, p.109-116, 1999. 